\title{
Multi-drug resistance in early and late onset neonatal sepsis in a tertiary hospital in Nigeria
}

\author{
Lateefat O. Sa'adu, ${ }^{1,2}$ Tope O. Obasa, ${ }^{3}$ Aishat O. Saka, ${ }^{3}$ Mohammed J. Saka, ${ }^{4}$ \\ Charles Nwabuisi ${ }^{1,5}$
}

${ }^{1}$ Department of Medical Microbiology and Parasitology, University of Ilorin Teaching Hospital; ${ }^{2}$ Assisted Reproductive Technology Unit, University of Ilorin Teaching Hospital; ${ }^{3}$ Department of Pediatric and Child Health, Faculty of Clinical Sciences, College of Health Sciences, University of Ilorin, Kwara State; ${ }^{4}$ Department of Epidemiology and Community Health, Faculty of Clinical Sciences, College of Health Sciences, University of Ilorin, Kwara State; ${ }^{5}$ Department of Medical Microbiology and Parasitology, Faculty of Basic Medical Sciences, College of Health Sciences, University of Ilorin, Kwara State, Nigeria

\begin{abstract}
Neonatal sepsis is a clinical syndrome characterized by systemic signs of infection, and accompanied by bacteremia in the first month of life and is responsible for $30-50 \%$ of total neonatal
\end{abstract}

Correspondence: Lateefat O. Sa'adu, Department of Medical Microbiology and Parasitology, University of Ilorin Teaching Hospital Kwara State; Assisted Reproductive Technology Unit, University of Ilorin Teaching Hospital Kwara State, Nigeria.

Tel. +2348038134711 .

E-mail: lateemayaki88@yahoo.com

Key words: Neonatal Sepsis, Early Onset sepsis, Late onset sepsis, Methicillin resistance Staphylococcus aureus, Extended spectrum beta lactamase.

Acknowledgments: We are highly grateful to the authors who have contributed to the funding, writing and review of this manuscript for publication. We are also grateful to the University of Ilorin Teaching hospital where the research work was carried out. We are also grateful to Mr. Oyekunle Lukman, an Intern with Medical microbiology and parasitology department of the same hospital, who assisted in laboratory analysis.

Contributions: LOS, TOO, CN conceived and designed the study; TOO collected blood samples; LOS performed laboratory analysis; LOS, AOS, MJS manuscript writing; LOS, TOO, AOS, MJS, CN manuscript reviewing and references search.

Conflict of interest: The authors declare no potential conflict of interest.

Funding: None.

Received for publication: 5 March 2019.

Revision received: 2 July 2019.

Accepted for publication: 31 July 2019.

This work is licensed under a Creative Commons Attribution NonCommercial 4.0 License (CC BY-NC 4.0).

CC Copyright: the Author(s), 2019

Licensee PAGEPress, Italy

Annals of African Medical Research 2019; 2:69

doi:10.4081/aamr.2019.69 deaths, each year in developing countries. This study investigated multi-drug resistant organisms associated with early and late onset neonatal sepsis in the University of Ilorin Teaching Hospital (UITH). It was a descriptive cross-sectional study. One hundred and sixty-two blood samples from neonates admitted into the neonatal intensive care unit of UITH with clinical diagnosis of sepsis were obtained. One milliliter of blood was taken per neonate and cultured aerobically in brain heart infusion broth and sub cultured onto blood and MacConkey agar plates. Identification of the isolates was carried out by colonial morphology, Gram stain microscopy and several biochemical tests. Antibiotic susceptibility test was done using the modified Kirby-Bauer method, screening for methicillin resistance staphylococcus aureus (MRSA) and extended spectrum beta lactamase (ESBL) was done by the cefoxiin-based methods and double disc synergy test respectively. Data analysis was carried out using Microsoft excel version 2007 and Epi-info version 2012. Sepsis was confirmed bacteriologically in $22.2 \%$ of the samples. The prevalence of multidrug resistant isolate was $29.0 \%$. The prevalence of MRSA was found to be $37 \%$ while that of ESBL producing Enterobacteria was $44.4 \%$ with ESBL producing Klebsiella pneumoniae and Escherichia coli prevalence of $50 \%$ and $25 \%$ respectively. This study shows a high prevalence of Methicillin Resistant Staphylococcus Aureus and Extended Spectrum Beta Lactamase producing Klebsiella pneumoniae and Escherichia coli causing neonatal sepsis in UITH Ilorin.

\section{Introduction}

Neonatal sepsis is a clinical syndrome characterized by systemic signs of infection, and accompanied by bacteremia in the first month of life. Sepsis is the most common cause of neonatal mortality, and is responsible for $30-50 \%$ of total neonatal deaths, each year in developing countries. ${ }^{1}$

Neonatal sepsis may be classified according to the time of onset of the disease: early onset (EOS) and late onset (LOS). The distinction has clinical relevance, as EOS is mainly due to bacteria acquired before and during delivery, and LOS is due to bacteria acquired after delivery (nosocomial or community sources). In the literature, however, there is little consensus as to what age limits apply. $^{2}$

Etiological causes of neonatal sepsis could be bacterial, viral, fungal or even toxin mediated. Both Gram negative and positive bacteria have been isolated from blood, and predominance of one type over the other varies from place to place and even in the same 
place over time. ${ }^{3}$ Bacteria commonly isolated in the samples included Klebsiella pneumoniae, Escherichia coli, Enterobacter species, Pseudomonas aeruginosa and Staphylococcus aureus..$^{3,4}$

Also, many multi-drug resistant (MDR) organisms have also been isolated such as Methicillin Resistant Staphylococcus aureus (MRSA) and Extended Spectrum Beta lactamases (ESBLs). 5,6

Methicillin resistance Staphylococcus aureus (MRSA) are strains of Staphylococcus aureus that are resistant to the isoxazoyl penicillins such as methicillin, oxacillin and flucloxacillin, the mechanism of resistance is as a result of an alteration in the target of the antibiotics. ${ }^{7}$ They were once confined largely to hospitals, other health care environments, and patients frequenting these facilities. Since the mid-1990s, however, there has been an explosion in the number of MRSA infections reported for populations lacking risk factors for exposure to the health care. This increase has been associated with the recognition of new MRSA strains, often called community-associated MRSA (CA-MRSA) strains, that have been responsible for a large proportion of the increased disease burden observed in the last decade. ${ }^{8}$

MRSA in Neonatal Intensive Care Units (NICU) has been reported and is commonly associated with episodic outbreaks from a single clone. Epidemics of MRSA infection have been associated with understaffing, overcrowding, improper cleaning of equipment and hands. ${ }^{9}$

Due to extensive use of $\beta$-lactam antibiotics over the last several decades in clinical practice, various $\beta$-lactamases have emerged. Extended-spectrum beta-lactamases (ESBLs) which were first reported in 1983, are mutant plasmid-mediated beta-lactamases derived from older, broad-spectrum beta-lactamases (e.g., TEM-1, TEM-2, SHV-1). These enzymes are most commonly produced by Klebsiella spp and Escherichia coli but may also occur in other Gram-negative bacteria, including Enterobacter, Salmonella, Proteus, Citrobacter spp, Morganella morganii, Serratia marcescens, Shigella dysenteriae, and Pseudomonas aeruginosa. ${ }^{10}$ They are inhibited by $\beta$-lactamase inhibitors such as clavulanic acid, sulbactam and tazobactam. The cephamycins i.e cefoxitin and cefotetan are resistant to the hydrolytic effect of these enzymes because of their methoxy group. The carbapenems i.e imipenem and meropenem are also not affected. ${ }^{11,12}$

The emergence of these superbugs (MRSA and ESBLs) poses a serious antibiotic management problem because of their resistance to multiple drugs and also these genes are easily transferred from one organism to the other via plasmids. This study therefore assesses the prevalence of MRSA and ESBLs among aerobic bacterial isolates of neonatal sepsis in University of Ilorin Teaching Hospital (UITH) Ilorin Kwara State Nigeria.

\section{Materials and Methods}

This descriptive cross-sectional study was conducted at the Neonatal Intensive Care Unit of the UITH. The Neonatal Intensive Care Unit of the hospital admits an average of 2000-2500 babies per annum, this comprises babies born within the hospital and those referred to the unit.

The study population included all neonate's new-born aged 028 days (Table 1) with clinical diagnosis of sepsis admitted into the Neonatal Intensive Care Unit of the Hospital from October 2013February 2014. Presence of any or a combination of the following signs and symptoms were used by the attending physician to establish a clinical diagnosis of neonatal sepsis: temperature instability, lethargy, irritability, mottling, pallor, petechiae, feeding intolerance, vomiting, diarrhea, abdominal distention, or respiratory dis- tress and neonates with risk factors for sepsis such as new-born of mothers with prolonged rupture of membrane (PROM) [rupture of membranes for more than 18 hours prior to delivery], maternal fever and or Vulval urinary or lower gastrointestinal infection before or during labor, foul smell of amniotic fluid and meconium stained fluid.

One milliliter of venous blood from a peripheral vein, was taken by resident doctors in the neonatal intensive care unit (NICU) under aseptic conditions, and before commencement of antibiotics, from 162 neonates. The blood was then transferred, using a new sterile needle through the rubber liner of the bottle cap, into the blood culture bottle containing $9 \mathrm{~mL}$ Brain Heart Infusion broth. A fresh ethanol-ether swab was used to wipe the top of each culture bottle and the tape was replaced. Each bottle was clearly labelled with the reference ID number of the neonate, and the date and time of collection. Samples were transferred to the microbiology laboratory of the University of Ilorin, Teaching Hospital within an hour of collection for further processing.

Samples were processed using the inoculated Brain Heart Infusion medium which was incubated at $37^{\circ} \mathrm{C}$ for 7 days and were examined daily for visible signs of bacterial growth. Growth was usually indicated by hemolysis of the red blood cells, gas bubbles in the medium, turbidity, or the appearance of small aggregates of bacterial growth in the broth, on the surface of the sedimented red cell (cotton balls) or occasionally along the walls of the bottle. Subcultures from blood cultures suspected of being positive were made on 5\% sheep blood agar and MacConkey agar. Strict aseptic technique was ensured to avoid contaminating the culture.

The blood and MacConkey agars were prepared according to the manufacturer's standard and one plate per batch of each of the agars was examined for sterility by incubating at $37^{\circ} \mathrm{C}$ for $24-48$ hours. The plates were then stored in the refrigerator, at a temperature of between $2^{\circ} \mathrm{C}$ to $10^{\circ} \mathrm{C}$ and used within seven days of production. The Blood agar and MacConkey agar plates were incubated at $37^{\circ} \mathrm{C}$ aerobically overnight. Bottles without evidence of growth were followed up by examining the broth daily, a final subculture was done at the end of 7 days or at appearance of turbidity, whichever occurred earlier.

Identification of isolates was carried out using standard laboratory procedures which includes colonial morphology, Gram stain microscopy and several biochemical tests as hemolytic activity on blood agar plates, catalase, coagulase (free and bound), DNase production, and growth on mannitol salt agar for Gram-positive isolates, and triple sugar iron (TSI), motility, indole, citrate utilization, urease, oxidase and hydrogen sulphide production and VogesProskauer (VP) test for Gram-negative bacilli as described by Cheesebrough and Forbes. ${ }^{13,14}$

\section{Antimicrobial susceptibility testing}

Antimicrobial susceptibility test was done using the modified Kirby-bauer method (CLSI 2012). ${ }^{15}$ For this study the following antibiotics were tested against the isolates following the procedures stated above: Gentamicinn $(10 \mu \mathrm{g})$, Ceftriaxone $(30 \mu \mathrm{g})$, Augmentin $(30 \mu \mathrm{g})$, Ceftazidime $(30 \mu \mathrm{g})$, Unasyn $(10 / 10 \mu \mathrm{g})$, Imipenem $(10 \mu \mathrm{g})$ and Vancomycin $(30 \mu \mathrm{g})$ while Staphylococcus aureus ATCC

Table 1. Age and sex distribution of neonates.

\begin{tabular}{lccc} 
Age (hours) & Female & Male & Total (\%) \\
Early onset sepsis $(<72)$ & 58 & 67 & $125(77.2)$ \\
Late onset sepsis $(\geq 72)$ & 12 & 25 & $37(22.8)$ \\
\hline Total (\%) & $70(43.2)$ & $92(56.8)$ & $162(100.0)$ \\
\hline
\end{tabular}


25923, Escherichia coli ATCC 25922, Pseudomonas aeruginosa ATCC 27853 were used as control strains.

\section{Methicillin resistance Staphylococcus Aureus}

The MRSA was detected by the Cefoxitin-based methods recommended in the guidelines of the Clinical Laboratory Standards Institute. (CLSI, 2012) (15 $^{15}$ All strains of Staphylococcus aureus were tested with $30 \mu \mathrm{g}$ cefoxitin discs (Mast, UK) on Mueller-Hinton agar plates. For each strain, the bacterial suspension was adjusted to 0.5 McFarlands standard. MRSA ATCC 3391 (Oxoid) and locally identified Methicillin sensitive Staphylococcus aureus (MSSA) were used as positive and negative controls respectively. The zone of inhibition was determined after 24 hours of incubation at $35^{\circ} \mathrm{C}$. Zone size was interpreted according to CLSI (2012) criteria: susceptible, $\geq 22 \mathrm{~mm}$; resistant, $\leq 21 \mathrm{~mm}$.

\section{Extended Spectrum Beta Lactamases}

According to CLSI guidelines, strains showing zone of inhibition of $\leq 22 \mathrm{~mm}$ for ceftazidime, $\leq 27 \mathrm{~mm}$ for cefotaxime, and $\leq 25 \mathrm{~mm}$ for ceftriaxone were selected for conformational tests for ESBL. The Double Disc Synergy Test (DSST) was used. The isolated colonies were inoculated in peptone water at $37^{\circ} \mathrm{C}$ for $2-6$ hour. The turbidity was adjusted to $0.5 \mathrm{Mc}$ Farlands standard and lawn culture was made on Mueller-Hinton agar using sterile swab, an Augmentin disc (20/10 $\mu \mathrm{g})$ was placed in the center of plate. On both sides of Augmentin disc, a disc of cefotaxime $(30 \mu \mathrm{g})$ and ceftazidime $(30 \mu \mathrm{g})$, was placed with center to center distance of $15 \mathrm{~mm}$ to the centrally placed disc (amoxicillin-clavulanic acid disc). The plate was incubated at $37^{\circ} \mathrm{C}$ for $16-18$ hours. ESBL production was interpreted as the $3 \mathrm{rd}-$ generation cephalosporin disc inhibition increases towards the Augmentin disc or if neither disc were inhibitory alone but bacterial growth was inhibited where the two antibiotics diffuses together. ${ }^{16}$

Data was entered into a computer and analyzed using Microsoft Excel version 2007 and Epi-info version 2012 software. Associations of categorical variables were tested using Chi square while statistical significance was set at $\mathrm{P}<0.05$. Results were presented in tables and charts.

\section{Results}

Majority (77.2\%) of the neonates recruited for the study were less than 72 hours i.e presenting with early onset sepsis (EOS), thus giving EOS: LOS ratio of 3.4:1.

Twenty three percent ( 36 blood samples) of the sample yielded positive bacterial growth. Two of these blood samples yielded more than one organism: in all, there were 38 isolates. The aerobic spore bearers were regarded as contaminants (Table 2).

Majority of the isolates (65.8\%) were from neonates less than 72 hours old which is EOS and this was not significant $\left(\chi^{2}=3.75\right.$, $\mathrm{P}=0.053)$. Gram negative organisms occurred more commonly as a cause of EOS (15 of 25 isolates) than Gram positive organisms (10 of 25 isolates), but this was not statistically significant $\left(\chi^{2}=0.22\right.$ and $\left.\mathrm{P}=0.63\right)$.

Eleven out of the thirty-eight isolates were multi-drug resistant thus giving a prevalence rate of $29.0 \%$ (Figure 1). Three out of the eight Staphylococcus aureus were Methicillin resistant giving MRSA prevalence rate of $37.5 \%$ (Figure 2). Out of the eighteen (18) Escherichia coli and Klebsiella pneumoniae isolated, eight (8) were ESBL producers, giving a prevalence rate of $44.4 \%$ (Figure 3 ). Half (50\%) of the Klebsiella pneumoniae isolated were ESBL producers.

\section{Discussion}

Neonatal sepsis remains an important cause of morbidity and mortality especially in developing countries, ${ }^{17}$ despite the increasing awareness of hospital infection control practices and introduction of new and more potent antimicrobial agents.

Our result showed a much higher occurrence of EOS compared to LOS. This is similar with report from Zhiling et al., ${ }^{18}$ where EOS is higher (66) than LOS (49). Consistently, early onset sepsis has been found to occur more frequently than late onset sepsis in neonates, and with a higher morbidity and mortality. ${ }^{18}$

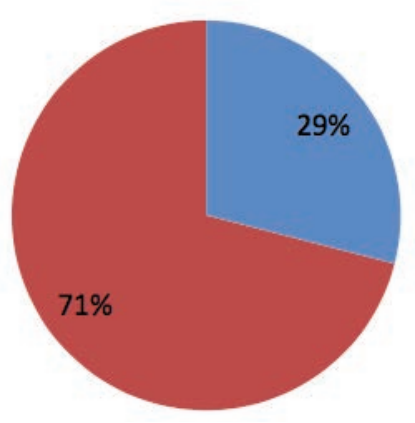

multi-drug resistant isolates

non multi drug resistant isolates

Figure 1. Prevalence of multi-drug resistant isolates.

Table 2. Distribution of isolates by age.

\begin{tabular}{|c|c|c|c|}
\hline Isolates & $\begin{array}{l}\text { Early onset sepsis } \\
\leq 72 \text { hrs }(\%)\end{array}$ & $\begin{array}{l}\text { Late onset sepsis } \\
\qquad 72 \text { hrs }(\%)\end{array}$ & Total (\%) \\
\hline $\begin{array}{l}\text { Gram negative } \\
\text { Escherichia coli } \\
\text { Klebsiella pneumoniae } \\
\text { Pseudomonas aeruginosa }\end{array}$ & $\begin{array}{l}3(75.0) \\
9(64.3) \\
3(100.0)\end{array}$ & $\begin{array}{l}1(25.0) \\
5(35.7) \\
0(0.0)\end{array}$ & $\begin{array}{l}4(100.0) \\
14(100.0) \\
3(100.0)\end{array}$ \\
\hline $\begin{array}{l}\text { Gram positive } \\
\text { Staphylococcus aureus } \\
\text { Staphylococcus saprophyticus } \\
\text { Enterococcus faecalis }\end{array}$ & $\begin{array}{l}3(37.5) \\
6(75.0) \\
1(100.0)\end{array}$ & $\begin{array}{c}5(62.5) \\
2(25.0) \\
0(0.0)\end{array}$ & $\begin{array}{l}8(100.0) \\
8(100.0) \\
1(100.0)\end{array}$ \\
\hline Total & $25(65.8)$ & $13(34.2)$ & $38(100.0)$ \\
\hline
\end{tabular}


Out of the 36 patients with positive blood culture, $65.8 \%$ had early onset sepsis (EOS) while $34.2 \%$ had late onset sepsis (LOS). This however contrasts with an earlier study by Mokuolu et al. ${ }^{4}$ in Ilorin where a higher preponderance of late onset sepsis $(73.6 \%)$ was reported.

Gram negative organisms were the predominant cause of early onset sepsis (60\%), with Klebsiella pneumoniae accounting for $60 \%$ and both Escherichia coli and Pseudomonas aeruginosa accounting for $20 \%$ each. This is quite similar with the findings of Mokuolu et al. ${ }^{4}$ where two-third of the early onset infections were due to Gram negative organisms with Klebsiella pneumoniae accounting for $57 \%$.

In the late onset category, Gram positive organisms predominated, accounting for 54\% with Staphylococcus aureus constituting $71.4 \%$ and Staphylococcus saprophyticus $28.6 \%$. This is also similar with the findings of Mokuolu et al., ${ }^{4}$ where Gram positive organisms also predominated in the late onset category and Staphylococcus aureus accounted for $38.8 \%$. These similarities may be due to the fact that the two studies were carried out in the same area hence, same prevailing strains.

The prevalence of MRSA as shown in Figure 2 is $37 \%$; this is similar to the findings of Ghandi et al., ${ }^{19}$ who reported MRSA prevalence of $31.25 \%$.It is however, higher than the findings of Prabhu et al., ${ }^{20}$ and Bhat et al., ${ }^{5}$ where MRSA prevalence of $29 \%$ and $23.07 \%$ were reported. This is also lower than MRSA prevalence of $50.94 \%, 66 \%$ and $66.7 \%$ reported by Singh et al., ${ }^{21}$ Karthikeyan et al.,22 and Hannan et al. ${ }^{23}$ The difference in prevalence may be due to the different prevailing strains, antibiotic prac-

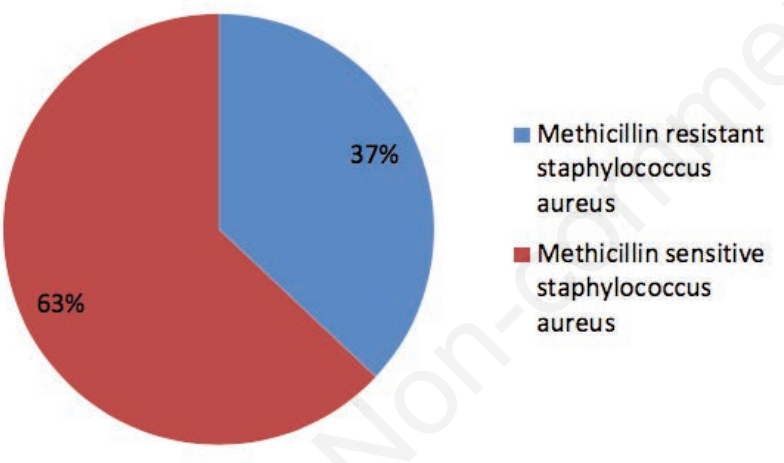

Figure 2. Methicillin sensitivity of Staphylococcus aureus.

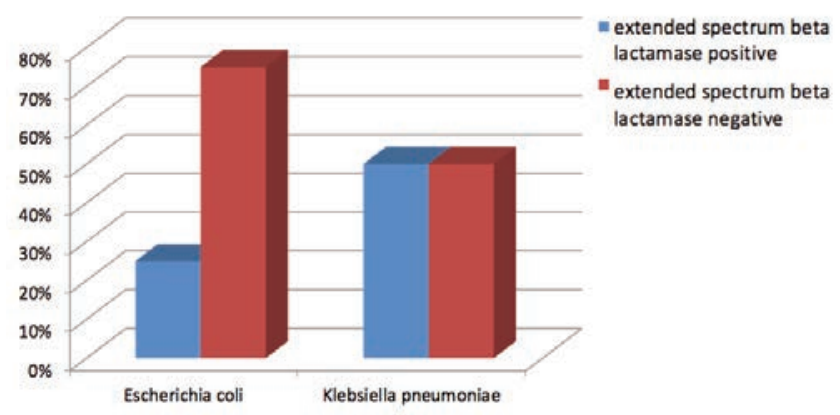

Figure 3. Extended spectrum beta-lactamase sensitivity among Escherichia coli and Klebsiella pneumoniae isolates. tices in these locations and also the screening method used; for this study the cefoxitin based method was used while some of the previous studies used the oxacillin method. Cefoxitin induces mecA gene better and gives a more accurate and reproducible results.

In this study extended spectrum beta-lactamase (ESBLs) prevalence of $44.4 \%$ was recorded among Klebsiella pneumoniae and Escherichia coli isolated (Figure 3). This is similar to the findings of Prabhu et al., ${ }^{20}$ and Bhat et al., ${ }^{5}$ where the prevalence of ESBL producing Gram negative bacilli were found to be $32 \%$ and $35 \%$ respectively. This is far lower than the reports of Shalini et al., ${ }^{6}$ and Dangre-Mundey et al., ${ }^{24}$ which reported ESBL prevalence of $72.3 \%$, and $95 \%$ among Gram negative bacilli. This may be due to different prevailing strains and antibiotic practices as the methodology is quite similar.

The prevalence of ESBL producers among Klebsiella pneumoniae was found to be $50 \%$, while $25 \%$ of the Escherichia coli isolated were ESBL producers. This is lower than the $80 \%$ and $63.6 \%$ prevalence of ESBL producing Klebsiella pneumoniae and Escherichia coli reported by Shalini et al., ${ }^{6}$ It is also lower than the $58 \%$ prevalence of ESBL producing Klebsiella pneumoniae reported by Jain et al., ${ }^{25}$ and $52.9 \%$ of ESBL producing Escherichia coli reported by Ghandi et al. ${ }^{19}$

\section{Conclusions}

This study shows a high prevalence of Methicillin Resistant Staphylococcus Aureus and Extended Spectrum Beta Lactamase producing Klebsiella pneumoniae and Escherichia coli causing neonatal sepsis in UITH Ilorin. MRSA and ESBL pose a challenge in the management of neonatal sepsis, they lead to an increase in the duration of hospital stay thereby increasing the cost of treatment. Also, by being multidrug resistant they increase mortality rate. We therefore recommend an effective hospital infection control program and periodic review of the hospital antibiotic policy.

\section{References}

1. Kale A, Jaybhaye D, Bonde V. Neonatal Sepsis: An update. Iran J Neonatol 2013;4:39-51.

2. Vergnano S, Sharland M, Kazembe P et al. Neonatal sepsis: An international perspective. 2005. Available from: www.archdischild.com. Accessed on: 8th August 2018.

3. Iregbu, KC, Elegba, OY, Babaniyi IB. Bacteriological profile of neonatal septicaemia in a tertiary hospital in Nigeria. Afr Health Sci 2006;6:151-4.

4. Mokuolu AO, Jiya N, Adesiyun OO. Neonatal Septicaemia in Ilorin: Bacterial Pathogens and Antibiotic Sensitivity Pattern. Afr J Med Sci 2002;31:127-30.

5. Bhat S, Kavita, Rao S. Bacteriology of Neonatal Septicaemia. JRRMS 2011;1:18-21.

6. Shalini A, Niranjan T, Balaji V, Atanu KJ. Prevalence of Extended-spectrum B-lactamase Producing Escherichia coli and Klebsiella spp in a Neonatal Intensive Care Unit. Indian Pediatr 2009;46:1106-7.

7. Baddour MM, Abuelkheir MM, Fatani AJ. Trends in antibiotic susceptibility patterns and epidemiology of MRSA isolates from several hospitals in Riyadh, Saudi Arabia. Ann Clin Microbiol Antimicrob 2006;5:30.

8. David ZM, Robert SD. Community-Associated MethicillinResistant Staphylococcus aureus: Epidemiology and Clinical Consequences of an Emerging Epidemic. Clin Microbiol Rev 
2010;23:616-87.

9. Kaufman D, Fairchild DK. Clinical Microbiology of Bacterial and Fungal sepsis in Very- Low-Birth- Weight infants. Clin Microbiol Rev 2004;17:638-80.

10. Kenneth ST. Controversies about Extended-Spectrum and AmpC beta-lactamases. Emerg Infect Dis 2001;7:333-5.

11. Ankur Goyal KN, Prasad AP, Sapna Gupta UG, Archana A. Extended spectrum beta-lactamase in Escherichia coli and Klebsiella pneumoniae \&associated risk factors. Indian J Med Res 2008;129:695-700.

12. Jonathan N. Screening for Extended-Spectrum Beta-lactamase-producing pathogenic Enterobacteria in District General Hospitals. J Clin Microbiol 2005;43:31488-90.

13. Cheesbrough M. Culturing Blood. District Laboratory practice in Tropical Countries. United Kindom, University Press, Cambridge. 2000. pp 124-30.

14. Forbes BA, Sahm DF, Weissfeld AS. Bloodstream Infections. Bailey and Scott's Diagnostic Microbiology, 12th Edition. Mosby Elsevier, China, 2007. pp 789-797.

15. CLSI. Performance standards for Antimicrobial disk susceptibility tests; Approved Standard, M02-A11, Clinical and laboratory Standard Institute 2012; vol.32, no.1, pp 9-13.

16. Vemula S, Vadde R. Prevalence of ESBL-producing Klebsiella pneumoniae isolates in tertiary care hospital. ISRN Microbiol 2011;2011:5.

17. Yelda AL, Álvarez-Nemegyei J, Velázquez RJ, et al. Risk factors and prognosis for neonatal sepsis in southern Mexico: analysis of a four-year historic cohort follow-up. BMC Pregnancy Child Health 2012;12:48.
18. Zhiling L, Zhijun X, Zhiping L, Qiao Z, et al. 116 cases of neonatal early-onset or late-onset sepsis: A single center retrospective analysis on pathogenic bacteria species distribution and antimicrobial susceptibility. Int J Clin Exp Med 2013;6:693-9.

19. Ghandi S, Ranjan K, Sapre N, Massani M. Incidence of neonatal sepsis in tertiary care hospital: An overview. Int J Med Sci Public Health 2013;2:162-475.

20. Prabhu K, Bhat S, Rao S. Bacteriologic profile and antibiogram of blood culture isolates in a pediatric care unit. J Lab Physicians 2010;2:85-8.

21. Singh S, Kumar S, Kumari P. Isolation of methicillin- resistant Staphylococcus aureus from neonatal sepsis at a tertiary care hospital. Int J Sci Stud 2019;6:25-8.

22. Karthikeyan G, Premkumar K. Neonatal Sepsis: Staphylococcus aureus as the predominant pathogen. Indian J Pediatr 2001;68:715-7.

23. Hannan A, Qamar MU, Usman M, et al. Multidrug resistant microrganisms causing neonatal septicaemia: In a tertiary hospital Lahore, Pakistan. Afr J Microbiol Res 2013;7:1896-902.

24. Dangre-Mudey G, Neelima S. Tankhiwale, Fule RP. Multidrug Resistance and Extended Spectrum Beta Lactamase Production in Klebsiella Species Isolated from Cases of Neonatal Septicaemia. J Life Sci 2012;4:59-62.

25. Jain A, Mondal R. Prevalence and antimicrobial resistance pattern of extended spectrum beta-lactamase producing Klebsiella spp isolated from cases of neonatal septicaemia. Indian J Med Res 2007;125:89-94. 